\title{
Inflammasome-driven arthritis: a new model of RA?
}

Should rheumatoid arthritis (RA) be considered a syndrome, rather than a single disease? In several widely used experimental models of arthritis, such as collagen-induced and antigen-induced arthritis, pathology occurs independently of the inflammasome. However, research now published in Nature describes an experimental model of the disease in which the Nlrp3 inflammasome has a prominent mechanistic role.

The investigators showed that mice lacking the RA-susceptibility gene Tnfaip3 (also known as A20) in myeloid cells spontaneously developed severe, erosive arthritis. Notably, mice deficient in both A20 and the IL- 1 receptor were protected from developing clinical and histopathologic signs of arthritis, demonstrating the importance of IL-1 signalling in this model.

Compared with A20-sufficient mice, $A 20^{\text {myel-KO }}$ mice showed excessive production of IL- $1 \beta$, IL- 6 and TNF.
Deletion of Nlrp3 or caspase-1 abrogated this increased secretion of IL- $1 \beta$ and IL-6, and also protected $A 20^{\text {myel-KO }}$ mice from arthritis pathology, including inflammation and bone erosion. Notably, TNF production was not affected by Nlrp3 or caspase-1 deletion.

"Mechanistically, we showed that A20 negatively regulates Nlrp3 inflammasome activation in myeloid cells," explains study leader Mohamed Lamkanfi. Stimulation of the Nlrp3 inflammasome in lipopolysaccharide (LPS)-primed A20deficient macrophages led to substantially increased caspase- 1 activation and secretion of IL-1 $\beta$; this hyperactivation was not observed in Nlrc4 or AIM2 inflammasomes. Furthermore, compared with wild-type macrophages, A20deficient cells showed higher expression levels of Nlrp 3 and proIL- $1 \beta$ at rest and after priming with LPS.

The investigators contend that the A20 ${ }^{\text {myel-KO }}$ model might have value in preclinical studies of therapies targeting the Nlrp3 inflammasome and IL-1 signalling. "There is clear evidence that disease in different mouse models of $\mathrm{RA}$ is driven by

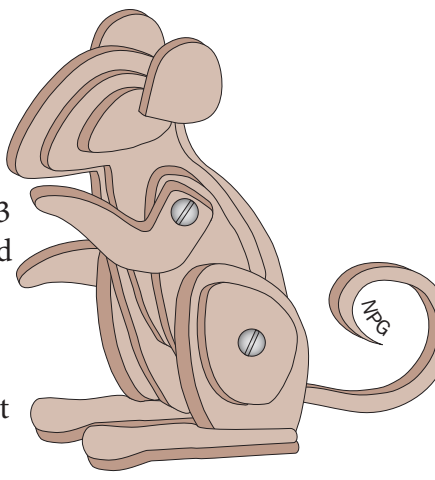
distinct immunological mechanisms," says Lamkanfi. "We believe something similar may apply in patients, and targeted studies in patients that correlate therapeutic responses to different therapies with their serological and/or genetic profiles may provide new and more effective treatment schemes."

Sarah Onuora

Original article Vande Walle, L. et al. Negative regulation of the NLRP3 inflammasome by A20 protects against arthritis. Nature doi:10.1038/nature13322 